\section{Repatriating the Canadian Thoracic Society annual general meeting}

Cor the past 10 years, the Canadian Thoracic Society (CTS) Annual General Meeting (AGM) has been held in conjunction with the American College of Chest Physicians' (ACCP) CHEST Conference in American cities, and every five years in Canada. This seemed perfectly normal to me and the CTS Executive Committee until the Canadian Respiratory Conference (CRC) forged its way to become the premiere national scientific meeting for the Canadian respiratory community. Surreptitiously, a nagging question presented itself: why is the CTS AGM usually being held in the United States when it could be held here in Canada in conjunction with the CRC?

The question may seem to be a 'no-brainer', but when you take into consideration the CTS' varied history, the answer is not that simple. The CTS is a national specialty society that took root in the Canadian Lung Association, formerly the Canadian Tuberculosis Association (CTA). Our first AGMs and scientific meetings were held in conjunction with CTA annual meetings. Later on, to better meet member needs, we chose to align our meetings with those of the Royal College of Physicians and Surgeons of Canada (RCPSC), with the RCPSC and the Canadian Society of Clinical Investigation, and the Canadian Medical Association. In 2000, we held our meeting with the American Thoracic Society International Conference in Toronto (Ontario) and, as of 2001, we chose to hold our meeting in conjunction with the ACCP CHEST Conference.

There was much debate every time we contemplated a change in the location of our AGM and scientific meetings. Would the new partnership meet our members' needs? Would it offer a strong scientific meeting that appealed to members? Did our members intend to attend the meeting/conference in the chosen location and in large numbers? How would moving the meeting affect our relationship with our partners?

The question came before us once again, but this time, the answer was clearer. The CTS is a national specialty society that could so evidently benefit from holding its AGM on Canadian soil. The CRC itself, which the Board has chosen as the new home for the AGM effective April 2012, will be held successively in all parts of Canada, and will be developed in collaboration with local provincial societies. Holding our AGM in conjunction with the CRC will help us foster collaboration with provincial thoracic societies and professionals from across Canada, and provide us with a venue to celebrate the vastness and richness of the Canadian respiratory community. Most of all, it will facilitate the participation of our members in the AGM, regardless of whether they travel to American conferences. Sooner or later, the CTS AGM may well be held in a town near you!

Although the AGM will move to Canada, the CTS will continue to hold an annual scientific program and the annual Christie Memorial Reception in conjunction with the CHEST Conference. We will continue to nurture our relationship with the ACCP, who has provided us with numerous benefits including a discount on conference registration and free meeting space. Although there will be no AGM at CHEST, we will still hold a members' forum to provide members with an opportunity to meet members of the Executive Committee and Board of Directors, and to share information at CHEST.

\section{Le rapatriement de l'assemblée générale annuelle de la Société canadienne de thoracologie}

Pepuis dix ans, l'assemblée générale annuelle (AGA) de la Société canadienne de thoracologie (SCT) a lieu dans des villes américaines, conjointement avec le congrès CHEST de l'American College of Chest Physicians (ACCP) qui, tous les cinq ans, est transféré au Canada. Ce fonctionnement nous semblait tout à fait normal, au comité directeur et à moi, jusqu'à ce que le Congrès canadien sur la santé respiratoire (CCSR) se fraie une place au point de devenir la principale rencontre scientifique nationale du milieu des soins respiratoires du Canada. Une question embêtante s'est mise à nous tarauder : pourquoi l'AGA de la SCT a-t-elle habituellement lieu aux ÉtatsUnis, alors qu'elle pourrait se dérouler ici, au Canada, conjointement avec le CCSR ?

De prime abord, la réponse peut sembler évidente, mais lorsqu'on tient compte de l'histoire diversifiée de la SCT, ce n'est plus aussi simple. La SCT est une société canadienne de spécialistes qui a pris racine au sein de l'Association pulmonaire du Canada, auparavant l'Association canadienne antituberculeuse (ACA). Les premières AGA et réunions scientifiques étaient organisées conjointement avec les réunions annuelles de l'ACA. Par la suite, afin de mieux répondre aux besoins des membres, les réunions ont été coordonnées avec celles du Collège royal des médecins et chirurgiens du Canada (CRMCC), du CRMCC et de la Société canadienne de recherches cliniques, puis de l'Association médicale canadienne. En 2000, les réunions se sont déroulées conjointement avec le congrès international de l'American Thoracic Society et, à compter de 2001, nous avons décidé de les faire coïncider avec le congrès CHEST de l'ACCP.

Chaque fois que nous envisagions de modifier l'emplacement de nos réunions, un débat animé éclatait. Le nouveau partenariat répondrait-il aux besoins des membres? Offrirait-il une réunion scientifique d'envergure qui plairait aux membres? Les membres seraient-ils nombreux à assister aux réunions et congrès à l'endroit proposé ? Quelles seraient les répercussions de ce changement sur nos relations avec nos partenaires?

La question s'est posée de nouveau, mais cette fois, la réponse était plus évidente. La SCT est une association nationale de spécialistes qui profiterait manifestement de tenir son AGA en sol canadien. Le CCSR, que le conseil d'administration de la SCT a choisi comme nouveau foyer de l'AGA à compter d'avril 2012, aura lieu successivement dans chaque région du Canada et sera élaboré en collaboration avec les associations pulmonaires provinciales locales. La tenue de l'AGA conjointement avec le CCSR favorisera la collaboration avec les sociétés de thoracologie provinciales et les professionnels de tout le Canada et offrira une tribune pour célébrer l'envergure et la richesse du milieu des soins respiratoires au pays. D'abord et avant tout, la participation des membres à l'AGA s'en trouvera facilitée, quelle que soit leur participation aux congrès américains. Tôt ou tard, l'AGA de la SCT pourrait bien avoir lieu près de chez vous !

L'AGA aura dorénavant lieu au Canada, mais la SCT tiendra toujours son programme scientifique annuel et sa réception commémorative Christie au congrès CHEST. Elle continuera d'entretenir sa relation avec l'ACCP, qui offre de nombreux avantages, tels qu'un rabais sur l'inscription au congrès et des salles de réunions gratuites. 
On a practical level, moving the CTS AGM to the CRC means that the terms of service of the current CTS Executive Committee will be extended until April 2012. Jean Bourbeau will continue to serve as secretary, Mark FitzGerald as treasurer, Robin McFadden as presidentelect, Denis O'Donnell as past-president and I as president.

Repatriating the AGM? It made a lot of sense to me, the Executive Committee, the Board of Directors and to most CTS members who responded to an online survey on that subject.

I hope to see many of you in Honolulu, Hawaii (USA) for CHEST 2011, and at the 2012 CRC in Vancouver, British Columbia!

Respectfully submitted, George A Fox MD MSc FRCPC FCCP President, Canadian Thoracic Society
Même s'il n'y aura pas d'AGA au congrès CHEST, nous proposerons tout de même une tribune qui permettra aux membres de rencontrer les membres du conseil d'administration et du comité directeur et de partager de l'information.

Sur le plan pratique, en raison du déménagement de l'AGA de la SCT au CCSR, le mandat des membres du comité directeur de la SCT sera prorogé jusqu'en avril 2012. Jean Bourbeau continuera d'assumer les fonctions de secrétaire, Mark FitzGerald, de trésorier, Rob McFadden, de président désigné, Denis O’Donnell, de président sortant, et moi, de président.

Rapatrier l'AGA ? Cela semblait tout à fait logique, tout autant pour moi que pour le comité directeur, le conseil d'administration et la plupart des membres de la SCT qui ont répondu à un sondage virtuel sur la question.

J'espère vous voir en grand nombre au congrès CHEST 2011 dans la ville d'Honolulu, à Hawaï (États-Unis), et au CCSR 2012, à Vancouver, en Colombie-Britannique!

Salutations distinguées George A Fox MD M. Sc. FRCPC FCCP Président, Société canadienne de thoracologie 


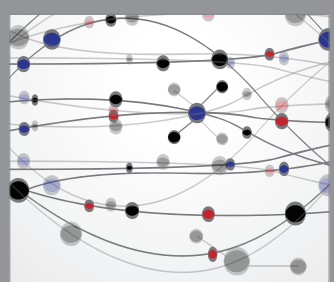

The Scientific World Journal
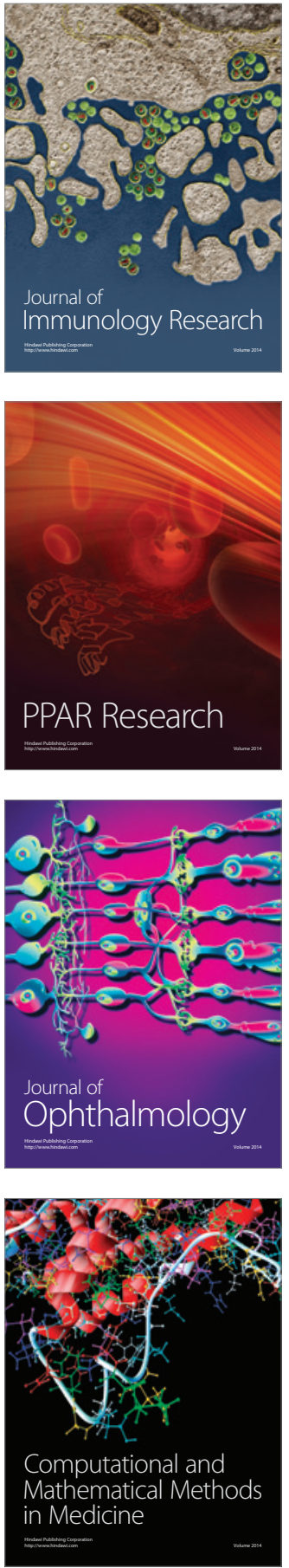

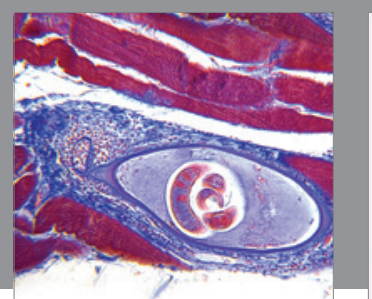

Gastroenterology Research and Practice

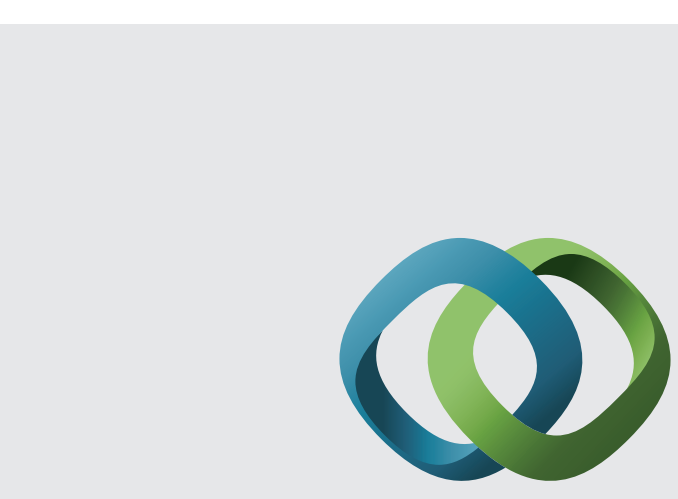

\section{Hindawi}

Submit your manuscripts at

http://www.hindawi.com
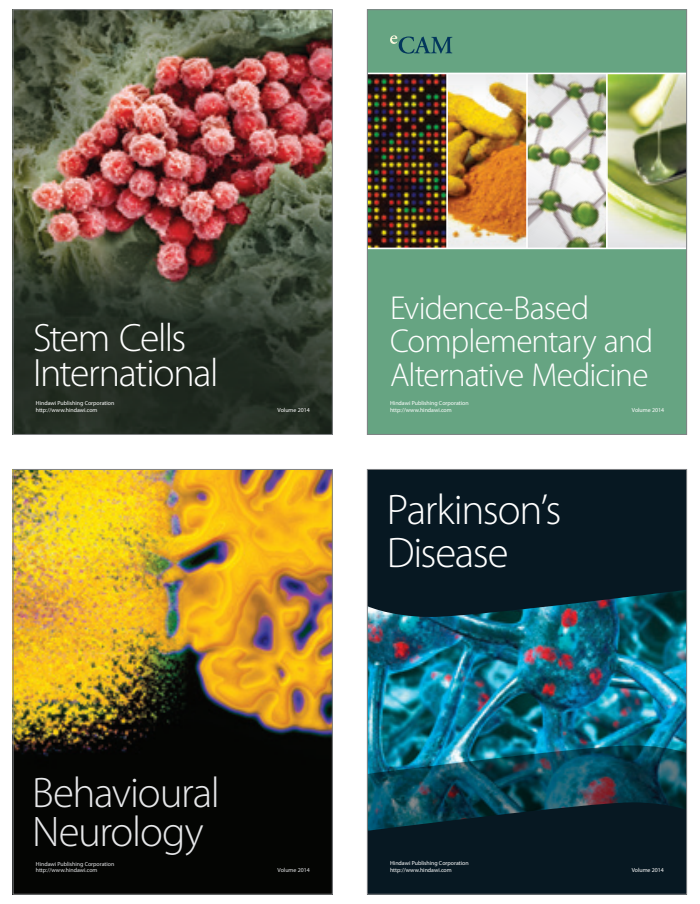
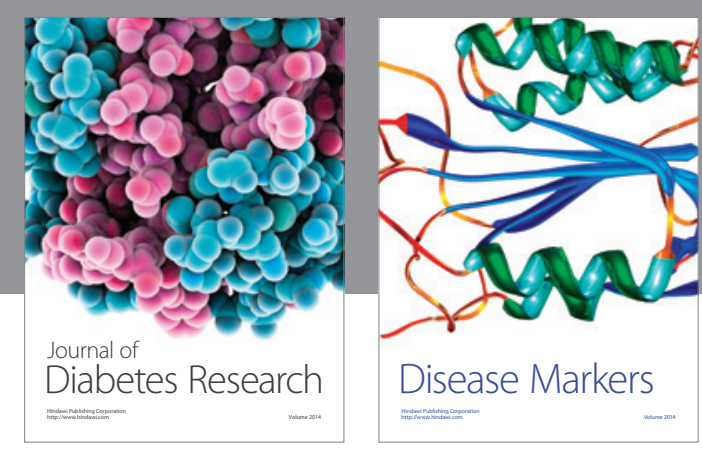

Disease Markers
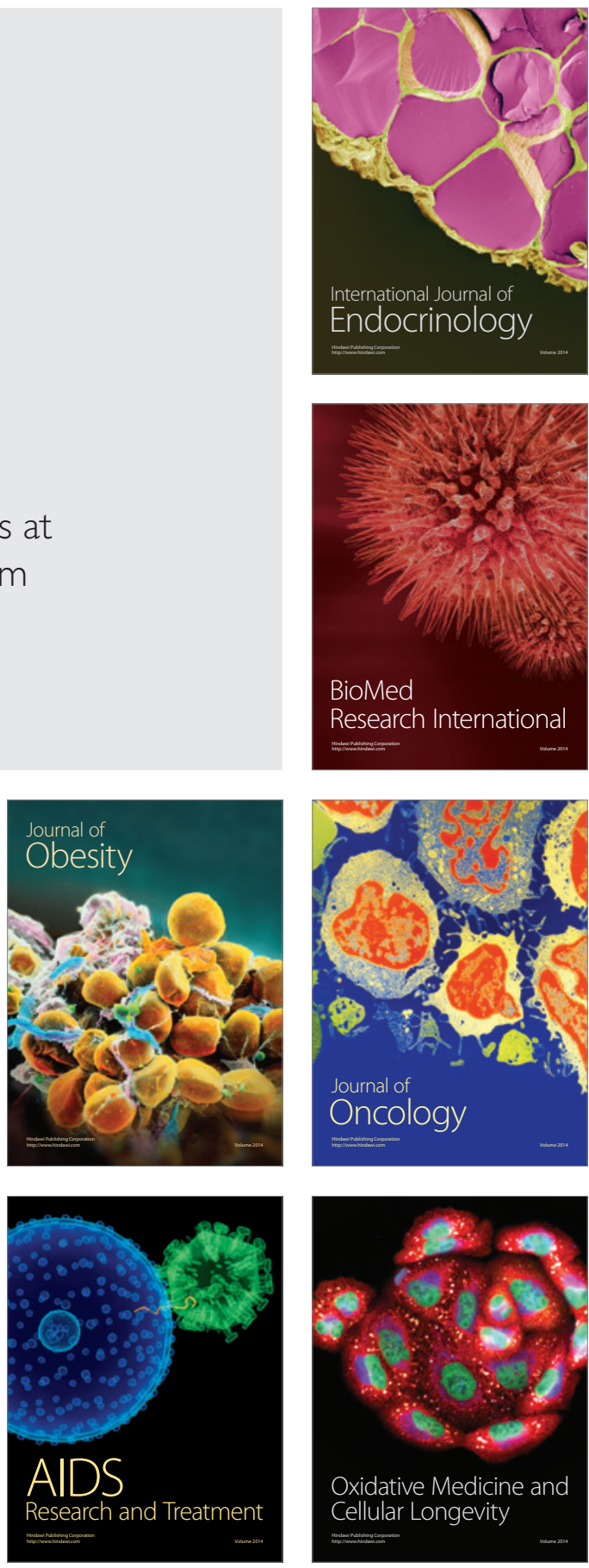